\title{
A Semiclassical Density Matrix Valid Beyond the Classically Allowed Region
}

\author{
M. Durand \\ Institut des Sciences Nucléaires (IN2 P3-U.S.M.G.), Grenoble, France \\ M. Brack and P. Schuck \\ Institut Laue Langevin, Grenoble, France \\ Received January 19, 1978
}

\begin{abstract}
We study semiclassical approximations to the density matrix of a system of Fermions in a one body potential. We derive an expression for the propagator in terms of first and second derivatives of the potential. Our result is thus exact for a harmonic oscillator potential and is equivalent to a partial resummation of the Wigner-Kirkwood h-expansion. It leads to densities which are well-defined also in the classically forbidden region.
\end{abstract}

\section{Introduction}

Semiclassical models based on a Wigner-Kirkwood type $\mathbf{h}$-expansion $[1-4]$ have recently regained interest in calculations of average binding energies of finite nuclei $[5,6]$. Although they have been shown to give correct averaged energies $[4,7]$, they suffer by the fact that they yield densities which are only defined inside the classically allowed region and diverge at the turning point. This defect can be traced back to the fact that the exact densities usually exhibit a $1 / \mathbf{h}$ dependence not allowing for a Taylor series expansion. *

The purpose of this investigation is to reconstruct from the so-called extended Thomas-Fermi expansion the original density as well as possible. We achieved a resummation by keeping only zeroth, first and second order derivatives of the potential. For the harmonic oscillator potential, our solution is exact.

Our work has been inspired by a recent paper of Bhaduri [8], who made an ad hoc ansatz for the singleparticle Bloch function, guided by its wellknown form for the harmonic oscillator. Our result shows that his ansatz correctly sums up the h-expansion including up to first order derivatives of the potential, but no consistent resummation of higher derivatives has been achieved.

Considering the exact density as a functional of the potential, our solution corresponds to a local harmoni-

\footnotetext{
$\star \mathbf{h}$ stands throughout this paper for $\hbar=h / 2 \pi$.
}

sation of the potential, putting higher than the second derivatives equal to zero. It turns out, that this is just a special case of an approach proposed recently by one of us [9], where the potential was developed around the classical path up to second order.

Our present method gives less accurate results (at least in the interior part of the nucleus), but it has the great advantage that one does not have to know the classical path. Instead, our expressions are entirely determined by the potential and its first and second derivatives. An important feature of our approach is that it can be generalized in a straightforward way to deformed three dimensional potentials.

\section{Expansion of the Propagator in Terms of Gradients of the Potential}

To obtain semiclassical expansions of the density matrix, it is convenient to study the single particle propagator (or Bloch density)

$$
C_{\mathbf{r}, \mathbf{r}^{\prime}}^{\beta}=\left\langle\mathbf{r}\left|e^{-\beta \hat{Q}}\right| \mathbf{r}^{\prime}\right\rangle,
$$

where $\beta=i t / \mathbf{h}$. The single particle density matrix is then given by an inverse Laplace transformation (see e.g. Ref. [10])

$\rho\left(\mathbf{r}, \mathbf{r}^{\prime}\right)=\mathscr{L}_{\mu}^{-1}\left[\frac{1}{\beta} C_{\mathbf{r}, \mathbf{r}^{\prime}}^{\beta}\right]$, 
where $\mu$ is the chemical potential. From (1) it is immediately seen that $C_{\mathbf{r}, \mathbf{r}^{\prime}}^{\beta}$ fulfills the Schrödinger (or Bloch) equation

$\frac{\partial}{\partial \beta} C_{\mathbf{r}, \mathbf{r}^{\prime}}^{\beta}+\int \hat{H}_{\mathbf{r}, \mathbf{r}^{\prime \prime}} C_{\mathbf{r}^{\prime \prime}, \mathbf{r}}^{\beta} d^{3} r^{\prime \prime}=0$.

We restrict ourselves presently to a local Hamiltonian

$\hat{H}_{\mathbf{r}, \mathbf{r}^{\prime}}=\hat{H}_{\mathbf{r}} \delta\left(\mathbf{r}-\mathbf{r}^{\prime}\right)=\left[-\frac{\mathbf{h}^{2}}{2 m} A_{r}+\boldsymbol{V}(\mathbf{r})\right] \delta\left(\mathbf{r}-\mathbf{r}^{\prime}\right)$.

It is convenient to introduce centre-of-mass and relative coordinates:

$\mathbf{q}=\frac{1}{2}\left(\mathbf{r}+\mathbf{r}^{\prime}\right), \quad \mathbf{s}=\mathbf{r}-\mathbf{r}^{\prime}$.

The exact solution of (3) is well-known for the free case $(V \equiv 0)$ :

$C_{\mathbf{q}, \mathbf{s}}^{\beta(\text { r re })}=\left(\frac{m}{2 \pi \mathbf{h}^{2}}\right)^{3 / 2} \beta^{-3 / 2} e^{-\frac{m}{2 \mathbf{h}^{2} \beta} \mathbf{s}^{2}}$

One also knows the exact solution for the harmonic oscillator potential [11]. In the spherical case, i.e.

$V(\mathbf{r})=\frac{1}{2} m \omega^{2} \mathbf{r}^{2}$,

one has

$$
\begin{aligned}
& C_{\mathbf{q}, \mathbf{s}}^{\beta(\mathrm{HO})}=\left(\frac{m}{2 \pi \mathbf{h}^{2}}\right)^{3 / 2}\left[\frac{\mathbf{h} \omega}{\operatorname{Sinh}(\beta \mathbf{h} \omega)}\right]^{3 / 2} \\
& \cdot \exp \left\{-\frac{m \omega}{\mathbf{h}}\left[\mathbf{q}^{2} \operatorname{Tgh}\left(\frac{\beta}{2} \mathbf{h} \omega\right)+\frac{\mathbf{s}^{2}}{4} \operatorname{Ctgh}\left(\frac{\beta}{2} \mathbf{h} \omega\right)\right]\right\} .
\end{aligned}
$$

The classical or Thomas-Fermi propagator is for a local potential given by

$C_{\mathbf{q}, \mathbf{s}}^{\beta(\mathrm{TF})}=C_{\mathbf{q}, \mathbf{s}}^{\beta(\mathrm{scc})} \cdot e^{-\beta \boldsymbol{V}(\mathbf{q})}$.

The Wigner-Kirkwood expansion $[1,2]$ of (1) is obtained by an ansatz in which the classical propagator (9) is multiplied by an unknown function which then is expanded in powers of $\mathbf{h}$.

To find a more general form of $C_{\mathbf{r}, \mathbf{r}^{\prime}}^{\beta}$ which includes both the classical (9) and the free limit (6), we make the following ansatz:

$C_{\mathbf{r}, \mathbf{r}^{\prime}}^{\beta}=\left(\frac{m}{2 \pi \mathbf{h}^{2}}\right)^{3 / 2} \frac{1}{\sqrt{G\left(\mathbf{r}, \mathbf{r}^{\prime} ; \beta\right)}} e^{-\boldsymbol{F}\left(\mathbf{r}, \mathbf{r}^{\prime} ; \beta\right)}$

This form suggests itself both from general WK.B-type functions, from the solution found in [9] and from the exact form of the harmonic oscillator (8).

Without loss of generality, we can assume both $F$ and $G$ in (10) to be odd functions of $\beta$. Inserting the ansatz (10) into the Bloch equation (3) and separating even and odd parts in $\beta$, we obtain the following coupled set of equations:

$$
\begin{aligned}
& \frac{\partial G}{\partial \beta}=\frac{\mathbf{h}^{2}}{m}\{G \Delta F-\boldsymbol{\nabla} F \cdot \nabla G\}, \\
& \frac{\partial F}{\partial \beta}=V-\frac{\mathbf{h}^{2}}{2 m}\left\{(\boldsymbol{\nabla} F)^{2}-\frac{1}{2 G} \Delta G+\frac{3}{4 G^{2}}(\boldsymbol{\nabla} G)^{2}\right\} .
\end{aligned}
$$

(The gradients act on one of the coordinates $\mathbf{r}, \mathbf{r}^{\prime}$ only.) In the 1-dimensional case, the equations retain exactly the same form, if the gradient $\boldsymbol{\nabla}$ is replaced by the first and the Laplacian $\Delta$ by the second derivative.

\subsection{One-Dimensional Case}

The exact solutions $F$ and $G$ of $(11,12)$ cannot be found in general-this would mean to solve the problem exactly. To find approximate solutions, one may expand the functions $F$ and $G$ in powers of $\beta$. Guided by the classical result (9) we write:

$$
\begin{aligned}
& F=\frac{f_{0}}{\beta}+f_{1} \beta+f_{3} \beta^{3}+\cdots, \\
& G=\beta+g_{3} \beta^{3}+g_{5} \beta^{5}+\cdots .
\end{aligned}
$$

(In three dimensions, the lowest term in $G$ would be $\beta^{3}$.) Inserting this ansatz into $(11,12)$ and comparing equal powers of $\beta$, one can get the coefficients $f_{n}$ and $g_{n}$ in $(13,14)$ as the solutions of simple linear first order differential equations. Since we started from equations which are not symmetric in $\mathbf{r}$ and $\mathbf{r}^{\prime}$ we have to explicitly symmetrize the results. (The propagator $C_{\mathbf{r}, \mathbf{r}^{\prime}}^{\beta}$ must be symmetric.) A more direct way to get symmetric solutions would be to start from the Wigner transform of the Bloch equation (3) [12]. This leads to the same results as the ones presented below.

The first few coefficients of the expansions $(13,14)$ are found to be

$$
\begin{aligned}
& f_{0}(q, s)=\frac{2 m}{\mathbf{h}^{2}} \frac{1}{4} s^{2}, \\
& f_{1}(q, s)=\frac{1}{s} \int_{0}^{s} \bar{V}\left(q, s^{\prime}\right) d s^{\prime}, \\
& g_{3}(q, s)=\frac{\mathbf{h}}{2 m} \frac{4}{s^{2}}\left[\bar{V}(q, s)-f_{1}(q, s)\right] \\
& f_{3}(q, s)=-\frac{\mathbf{h}^{2}}{2 m} \frac{1}{s^{3}}\left\{\int_{0}^{s}\left[\bar{V}\left(q, s^{\prime}\right)-f_{1}\left(q, s^{\prime}\right)\right]^{2} d s^{\prime}\right. \\
& \left.+\int_{0}^{s}\left[V V\left(q, s^{\prime}\right)\right]^{2} d s^{\prime}\right\}+\mathcal{O}\left(\mathbf{h}^{4}\right) .
\end{aligned}
$$


Hereby we have defined

$$
\begin{aligned}
& \bar{V}(q, s)=\frac{1}{2}\left[V(x)+V\left(x^{\prime}\right)\right] \\
& =\frac{1}{2}\left[V\left(q+\frac{s}{2}\right)+V\left(q-\frac{s}{2}\right)\right], \\
& V V(q, s)=\frac{1}{2}\left[V(x)-V\left(x^{\prime}\right)\right] \\
& =\frac{1}{2}\left[V\left(q+\frac{s}{2}\right)-V\left(q-\frac{s}{2}\right)\right] .
\end{aligned}
$$

It makes sense to expand these functions in powers of $s$ :

$$
\begin{aligned}
& f_{1}(q, s)=V(q)+\frac{1}{24} s^{2} V^{\prime \prime}(q)+\mathcal{O}\left(s^{4}\right) . \\
& f_{3}(q, s)=-\frac{\mathbf{h}^{2}}{2 m}\left\{\frac{1}{12}\left[V^{\prime}(q)\right]^{2}\right. \\
& \left.+\frac{1}{720} s^{2}\left[V^{\prime \prime}(q)\right]^{2}+\mathcal{O}\left(s^{4}\right)\right\}+\mathcal{O}\left(\mathbf{h}^{4}\right), \\
& g_{3}(q, s)=\frac{\mathbf{h}^{2}}{2 m}\left\{\frac{1}{3} V^{\prime \prime}(q)+\frac{1}{120} s^{2} V^{(\mathbf{I V})}(q)+\mathcal{O}\left(s^{4}\right)\right\} .
\end{aligned}
$$

We see that neglecting all the derivatives of the potential $V$ leads to the (1-dimensional) Thomas-Fermi result:

$C_{q, s}^{\beta(T F)}=\left(\frac{m}{2 \pi \mathbf{h}^{2}}\right)^{1 / 2} \beta^{-1 / 2} \cdot e^{-\frac{m}{2 \mathbf{h}^{2} \beta} s^{2}} \cdot e^{-\beta V(q)}$.

Our solution for $f_{1}(16,21)$ shows that it is not correct up to order $s^{2}$, if one replaces $V(q)$ in the TF-solution (24) by the average potential $\bar{V}(q, s)(19)$ [13]. Indeed, this would not give the correct result for the harmonic oscillator. By expanding the exponent in (8) to order $\beta$, one can easily check that $f_{1}(21)$ has the right coefficient $\left(\frac{1}{24}\right)$ of the second order term, whereas $\bar{V}(19)$ would give a wrong coefficient $\left(\frac{1}{8}\right)$.

The above results show that our approach to the exact propagator is given as a function of $V$ and all its derivatives. Neglecting all the derivatives yields the Thomas-Fermi solution. We now define an approximation scheme where we keep derivatives of $V$ up to a certain order, neglect all the higher ones and try to sum up all terms in the series for $F(13)$ and $G(14)$ which then remain. In this way we obtain a series of successive approximations which may yield improved solutions for the propagator $C_{q, s}^{\beta}$.

Keeping the first derivative of $V$ gives only one extra contribution from $f_{3}(22)$, and no further summation is needed. This gives what we may call the "linearized" approximation to the propagator (which is exact for a linear potential):

$$
C_{q, s}^{\beta(1)}=\left(\frac{m}{2 \pi \mathbf{h}^{2}}\right)^{1 / 2} \beta^{-1 / 2} \cdot e^{-\frac{m}{2 \mathbf{h}^{2} \beta} s^{2}} \cdot e^{-\beta V(q)+\frac{\mathbf{h}^{2}}{24 m} \beta^{3}\left[V^{\prime}(q)\right]^{2}} .
$$

The form (25) can easily be Laplace inverted analytically and leads to a folding of the Thomas-Fermi density with an Airy function (see below).

Including higher order derivatives of $V$ leads to an infinite number of terms both in $F$ and $G$. One notes, however, that if one puts all third and higher derivatives of $V$ equal to zero, then also all the derivatives of $G$ can be neglected (since $G$ is in lowest order, equation (23), proportional to $\left.V^{\prime \prime}\right)$; similarly, the third and higher derivatives of $F$ can then be neglected. To this order, the coupled system of equations for $F$ and $G$ then reduces to (in the one-dimensional case):

$$
\begin{aligned}
& \frac{\partial G}{\partial \beta}=\frac{\mathbf{h}^{2}}{m} G F^{\prime \prime}, \\
& \frac{\partial F}{\partial \beta}=V-\frac{\mathbf{h}^{2}}{2 m}\left(F^{\prime}\right)^{2} .
\end{aligned}
$$

(Here the prime denotes differentiation with respect to $x$.)

These equations can now easily be solved to get $G$ in a closed form. From (26) one gets directly $F$ in terms of $G$ by integrating twice. Eliminating $F$ in this way and taking suitable derivatives of both equations, one arrives at the equation

$\frac{\partial^{2} G}{\partial \beta^{2}}=\frac{\mathbf{h}^{2}}{m} V^{\prime \prime} G$

which has the solution

$G^{(2)}=\left[\frac{\mathbf{h}^{2}}{m} V^{\prime \prime}(q)\right]^{-1 / 2} \operatorname{Sinh}\left(\left[\frac{\mathbf{h}^{2}}{m} \boldsymbol{V}^{\prime \prime}(q)\right]^{1 / 2} \beta\right)$.

The solution of $F$, which in principle now is given through (26), is not easy to find in a symmetrized form and with the right boundary properties. However, working out the series expansion for $F$ (13) with the truncated equations $(26,27)$ and summing it up, one finds the solution

$$
\begin{aligned}
& F^{(2)}=\beta\left[V(q)-\frac{\left[V^{\prime}(q)\right]^{2}}{2 V^{\prime \prime}(q)}\right] \\
& +\frac{m}{\mathbf{h}^{2}} \sqrt{\frac{\mathbf{h}^{2}}{m} V^{\prime \prime}(q)}\left[\frac{V^{\prime}(q)}{V^{\prime \prime}(q)}\right]^{2} \operatorname{Tgh}\left(\frac{\beta}{2} \sqrt{\frac{\mathbf{h}^{2}}{m} V^{\prime \prime}(q)}\right) \\
& +\frac{m}{4 \mathbf{h}^{2}} s^{2} \sqrt{\frac{\mathbf{h}^{2}}{m} V^{\prime \prime}(q)} \operatorname{Ctgh}\left(\frac{\beta}{2} \sqrt{\left.\frac{\mathbf{h}^{2}}{m} V^{\prime \prime}(q)\right)} .\right.
\end{aligned}
$$

Equations (29) and (30) hold for any value of $V^{\prime \prime}(q)$. If $V^{\prime \prime}(q)<0$, the hyperbolic functions go over into the corresponding circular functions and $F^{(2)}$ and $G^{(2)}$ stay real. For $V^{\prime \prime}(q)=0$ one obtains the "linearized" solution (25). (Note that all derivatives in $(29,30)$ are with respect to $q$ !) 
It is easy to verify, that the solutions $(29,30)$ satisfy the coupled equations $(26,27)$ if one neglects higher than second derivatives of $V$. We note that the non-local part of $F^{(2)}$ is proportional to $s^{2}$, whereas $G^{(2)}$ is purely local up to this order. In fact, all terms of order $s^{4}$ or higher in the expansion (21)-(23) contain higher than second derivatives of $V$. Thus, expanding the potential up to second order and retaining quadratic terms in the nonlocality is consistent.

The "harmonized" approximation for the propagator which we thus obtain

$C_{q, s}^{\beta(2)}=\left(\frac{m}{2 \pi \mathbf{h}^{2}}\right)^{1 / 2} \frac{1}{\sqrt{G^{(2)}}} e^{-F^{(2)}}$,

has all the required boundary properties. It is exact for the harmonic oscillator potential, as can easily be checked by comparing to the 1-dimensional equivalent of (8). For anharmonic potentials we see that the quantity

$d=\sqrt{\frac{\mathbf{h}^{2}}{m}\left|V^{\prime \prime}(q)\right|}$

plays the role of a "local" oscillator frequency, whereas

$\eta=\frac{V^{\prime}(q)}{V^{\prime \prime}(q)}$

is a "generalized" centre-of-mass coordinate. Thus, the result (31) is equivalent to a locally harmonic approximation of the Schrödinger equation (3).

We shall show in Section 3 below that our result (31) can also be viewed as a partial resummation of the Wigner-Kirkwood expansion.

We now have to Laplace invert the propagator (31) in order to obtain the density matrix. This can be done analytically using "Mehler's" formulae [14]. For the case $V^{\prime \prime}>0$ one obtains

$$
\begin{aligned}
& \rho^{(2)}(q, s)=\sqrt{\lambda} e^{-\frac{\lambda}{2}\left(\xi^{2}+\xi^{\prime 2}\right)} \sum_{n=0}^{\infty} \mathbf{H}_{n}(\sqrt{\lambda} \xi) \mathbf{H}_{n}\left(\sqrt{\lambda} \xi^{\prime}\right) \\
& \cdot \Theta\left[\mu(q)-d\left(n+\frac{1}{2}\right)\right] \quad\left(V^{\prime \prime}(q)>0\right),
\end{aligned}
$$

where $\mathbf{H}_{n}$ are the normalized Hermite polynomials and the following definitions are used:

$$
\begin{aligned}
& \lambda=\frac{m}{\mathbf{h}^{2}} d=\sqrt{\frac{m}{\mathbf{h}^{2}}}\left|V^{\prime \prime}(q)\right|, \\
& \xi=\eta+\frac{1}{2} s, \quad \xi^{\prime}=\eta-\frac{1}{2} s, \\
& \mu(q)=\mu-V(q)+\frac{1}{2} \eta V^{\prime}(q) .
\end{aligned}
$$

For the case $V^{\prime \prime}<0$ one obtains similarly

$$
\begin{aligned}
& \rho^{(2)}(q, s)=\frac{\sqrt{2 \lambda}}{4 \pi d} \int_{-\infty}^{\mu(q)} \frac{e^{\frac{\pi E}{2 d}}}{\sin (-\pi v)}\left\{D_{v}\left(\sqrt{2 \lambda} e^{-\frac{i \pi}{4}} \xi\right)\right. \\
& \cdot D_{-v-1}\left(\sqrt{2 \lambda} e^{\frac{i \pi}{4}} \xi^{\prime}\right)+D_{v}\left(-\sqrt{2 \lambda} e^{-\frac{i \pi}{4}} \xi\right) \\
& \left.\cdot D_{-v-1}\left(-\sqrt{2 \lambda} e^{\frac{i \pi}{4}} \xi^{\prime}\right)\right\} d E \quad\left(V^{\prime \prime}(q)<0\right) .
\end{aligned}
$$

Here $D_{v}(z)$ are the parabolic cylinder functions and the index $v$ is defined by

$v=i \frac{E}{d}-\frac{1}{2}$.

For a harmonic oscillator potential, equation (34) reduces trivially to the exact density matrix obtained by filling the states below the Fermi level $\mu$. For anharmonic potentials, $\rho^{(2)}(34)$ has the unpleasant feature to show discontinuities at certain points $q$. This is due to the fact that the "local spectrum"

$d\left(n+\frac{1}{2}\right)+V-\frac{1}{2} \eta V^{\prime}=\varepsilon_{n}(q)$

becomes position-dependent. We see thus that our improved quantum mechanical treatment leads to spurious quantum fluctuations. These local fluctuations can however not reproduce the true density oscillations which are global effects. In order to average out these local fluctuations in a consistent way, we applied in our numerical calculations (see Sect. 4 below) a local Strutinsky-smoothing [15] which is known to give well-defined averaged harmonic-oscillator densities $[4,16]$.

The most important feature of our "harmonized" solution is that it leads to densities which are welldefined at the classical turning point and have the correct exponential fall-off in the outside of the surface. In the case $V^{\prime \prime}(q)=0$, the density reduces to the Laplace inversed of the "linearized" propagator (25):

$$
\begin{aligned}
& \rho^{(1)}(q, s)=\sqrt{\frac{2 m}{\mathbf{h}^{2}} \sigma(q)} \int_{-\infty}^{\mu} \mathbf{A i}^{2}[-\sigma(q)(E-V(q))] \\
& \mathbf{J}_{0}\left(s \sqrt{\frac{2 m}{\mathbf{h}^{2}}(\mu-E)}\right) d E
\end{aligned}
$$

where

$\sigma(q)=\left(\frac{2 m}{\mathbf{h}^{2}}\right)^{1 / 3} \cdot\left[V^{\prime}(q)\right]^{-2 / 3} \quad\left(V^{\prime}(q) \neq 0\right)$.

In (41), $\mathbf{A i}(x)$ is the Airy function (e.g. [10]) and $\mathbf{J}_{0}(x)$ the zeroth order Bessel function. An alternative form of $\rho^{(1)}(41)$ is found using the integral representation of the Airy function and reads 


$$
\begin{aligned}
& \rho^{(1)}(q, s)=2^{2 / 3} \sigma(q) \int_{-\infty}^{\mu} \rho^{(\mathrm{TF})}(q, s ; \mu-E) \\
& \mathbf{A i}\left[-2^{2 / 3} \sigma(q) E\right] d E .
\end{aligned}
$$

This form shows that $\rho^{(1)}$ is a convolution of the Thomas-Fermi density $\rho^{\text {(TF) }}(q, s ; \mu)$ with an Airy function, where

$\rho^{(\mathrm{TF})}(q, s ; \mu)=\frac{1}{\pi} k_{\mu}(q) j_{0}\left(s k_{\mu}(q)\right)$.

Here the local Fermi momentum is

$k_{E}(q)=\sqrt{\frac{2 m}{\mathbf{h}^{2}}(E-V(q))}$,

and $j_{0}(x)$ in (44) is the zeroth order spherical Bessel function. For $V^{\prime} \rightarrow 0$, the Airy function in (43) goes over into a delta function and $\rho^{(1)}$ reduces to the ThomasFermi density (44).

In all the above results, the chemical potential $\mu$ is determined by normalizing the local density $\rho(q, s=0)$ to the particle number.

\subsection{Three-dimensional Case}

The extension of our approach to three dimensions is in principle straightforward. Equations (11),(12) are again solved by expansions like in (13) and (14); in the latter, the lowest term is now $\beta^{3}$.

Since we in our numerical Section 4 will only present a few preliminary results for a spherical 3-dimensional potential, we restrict ourselves here to a short presentation of the formulae. A more detailed elaboration on the three-dimensional case, including deformations, will be given in a forthcoming paper.

In the "linearized" case, where all second and higher derivatives of the potential $V(\mathbf{r})$ are neglected, the generalization of the result (25) is found without any problems:

$$
C_{\mathbf{q}, \mathbf{s}}^{\beta(1)}=\left(\frac{m}{2 \pi \mathbf{h}^{2}}\right)^{3 / 2} \beta^{-3 / 2} e^{-\frac{m}{2 \mathbf{h}^{2} \beta} \mathbf{s}^{2}} e^{-\beta V(\mathbf{q})+\frac{\mathbf{h}^{2}}{24 m} \beta^{3}} \mid \boldsymbol{\nabla}^{V(\mathbf{q})]^{2}}
$$

(Note that here again, the gradient in $\boldsymbol{V} V$ acts on the c.$m$. coordinate $q !$ ). The density matrix obtained by Laplace inverting (46) is again given by (43), the Thomas-Fermi solution this time being (including a spin factor 2)

$$
\rho^{(\mathrm{TF})}(\mathbf{q}, \mathbf{s})=\frac{1}{3 \pi^{2}} k_{\mu}^{3}(\mathbf{q}) \frac{3}{|\mathbf{s}| k_{\mu}} j_{1}\left(|\mathbf{s}| k_{\mu}\right)
$$

(The Fermi-momentum $k_{\mu}$ is given in (45); in the quantity $\sigma(q)(42)$, the derivative $V^{\prime}(q)$ has to be replaced by the gradient $\boldsymbol{V} V(\mathbf{q})$.)

The "harmonized" approximation looks at first much more complicated; a careful book-keeping of all six diagonal and mixed second derivatives in the expansions of $F$ and $G$ is required. The problem is however simplified if one introduces local normal coordinates. In fact, it is clear that for a separable potential $V(x, y, z)=V_{1}(x)+V_{2}(y)+V_{3}(z)$, the generalization of the one-dimensional result (31) of $C_{q, s}^{\beta(2)}$ is just a product

$C_{\mathbf{q}, \mathbf{s}}^{\beta(2)}=\prod_{i=1}^{3} C_{i}\left(q_{i}, s_{i}\right)$

where each of the $C_{i}$ is given by (31), replacing $V$ by $V_{i}$ and the derivatives $V^{\prime}, V^{\prime \prime}$ by the corresponding partial derivatives with respect to $q_{i}$.

Now, in the approximation where we neglect higher than second derivatives of $V$, we can always locally separate the potential by introducing the local normal coordinates. These are found by diagonalizing the tensor $V_{i j}$ of the partial second derivatives (with respect to the c.-m. coordinates)

$\sum_{k} V_{i k} a_{k j}=\lambda_{i} a_{i j} \quad(i, j, k=1,2,3)$,

where

$V_{i k}=\frac{\partial^{2} V(\mathbf{q})}{\partial q_{i} \partial q_{k}}$.

In terms of the normalized eigenvectors $a_{i j}$ of (49), the generalized local c.-m. coordinates $\eta_{i}$ (cf. Eq. (33)) are then given by

$\eta_{i}=\sum_{j=1}^{3} a_{j i} d_{j}$

where

$d_{i}=\frac{D_{i}}{D} ; \quad D=\operatorname{det}\left|V_{i j}\right|$,

and $D_{i}$ is the determinant of $V_{i j}(50)$ after replacing the $i$ th column by $V_{i}=\frac{\partial V}{\partial q_{i}}(\mathbf{q})$. The local normal frequencies (cf. Eq. (32)) are then given by the eigenvalues $\lambda_{i}$ of (49):

$\omega_{i}=\sqrt{\frac{\lambda_{i}}{m}}$

Note that all these quantities, and thus particularly also the $\eta_{i}$ and $\omega_{i}$, are functions of the c.-m. coordinate q. In the one-dimensional case, the above definitions lead back to $(32,33)$, as is easily checked. 
In terms of the above expressions, the "harmonized" approximation to the propagator in three dimensions now becomes

$C_{\mathbf{q}, \mathbf{s}}^{\beta(2)}=e^{-\beta \tilde{V}(\mathbf{q})} \prod_{i=1}^{3} C_{i}\left(\eta_{i}, s_{i}\right)$,

where

$\tilde{V}(\mathbf{q})=V(\mathbf{q})-\frac{1}{2 D} \sum_{i=1}^{3} V_{i} D_{i}$,

and

$$
\begin{aligned}
& C_{i}\left(\eta_{i}, s_{i}\right)=\left(\frac{m}{2 \pi \mathbf{h}^{2}}\right)^{1 / 2}\left[\frac{\mathbf{h} \omega_{i}}{\operatorname{Sinh}\left(\mathbf{h} \omega_{i} \beta\right)}\right]^{1 / 2} \\
& \cdot \exp \left\{-\frac{m \omega_{i}}{\mathbf{h}}\left[\eta_{i}^{2} \operatorname{Tgh}\left(\frac{\beta}{2} \mathbf{h} \omega_{i}\right)+\frac{1}{4} s_{i}^{2} \operatorname{Ctgh}\left(\frac{\beta}{2} \mathbf{h} \omega_{i}\right)\right]\right\} .
\end{aligned}
$$

(Note that the relative coordinates remain unchanged: $\mathbf{s}=\mathbf{r}-\mathbf{r}^{\prime}$.)

For a harmonic oscillator potential $\eta_{i} \equiv q_{i}$ and the propagator (54) reduces to the expression (8) for the spherical case.

The inverse Laplace transform of the propagator (54) is done in the same way as shown in the one-dimensional case above. Since in our numerical calculations, we did not program the direct expressions for the densities $\rho(\mathbf{r})$, but performed the Laplace inversion by a numerical integration, we do not give here their explicit expressions which are rather lengthy depending on the signs of the local curvatures $\lambda_{i}$. (If all three $\lambda_{i}$ are positive, the density is just a triple sum of squared Hermite functions in the three normal coordinates $\xi_{i}, \xi_{i}^{\prime}$ $=\eta_{i} \pm \frac{1}{2} s_{i}$; cf. Eq. (34).)

\subsection{Kinetic Energy Density and Total Energy for a Local one Body Potential}

Within the Hartree-Fock approximation, the total nuclear binding energy is entirely determined by the effective nucleon-nucleon interaction and the density matrix $\rho\left(\mathbf{r}, \mathbf{r}^{\prime}\right)$. For effective interactions of the Skyrmetype [17] it is however possible to express the energy as a three-dimensional integral over local densities only which are derived from the density matrix. For a case without spin-orbit interaction, the only other quantity which one needs to know besides the density $\rho(\mathbf{r})$ is the kinetic energy density defined by

$\tau(\mathbf{r})=\left.\frac{\mathbf{h}^{2}}{2 m} \boldsymbol{V}_{\boldsymbol{r}^{\prime}} \cdot \boldsymbol{V}_{\boldsymbol{r}^{\prime}} \rho\left(\mathbf{r}, \mathbf{r}^{\prime}\right)\right|_{\mathbf{r}=\mathbf{r}^{\prime}}$.

The function $\tau(\mathbf{r})$ appears also in the potential energy for Skyrme interactions [17].
In the present work, we do not want to calculate selfconsistent potentials and energies, but we limit ourselves to the case of a velocity independent local one body potential $V(\mathbf{r})$. The total energy, corresponding to the sum of occupied levels, is then given by

$E=\int d^{3} r\{\tau(\mathbf{r})+V(\mathbf{r}) \rho(\mathbf{r})\}$.

Apart from a divergence whose integral is zero, the density $\tau(\mathbf{r})$ is then equal to

$\tau(\mathbf{r})=-\left.\frac{\mathbf{h}^{2}}{2 m} \Delta_{\mathbf{r}} \rho\left(\mathbf{r}, \mathbf{r}^{\prime}\right)\right|_{\mathbf{r}=\mathbf{r}^{\prime}}=\mathscr{L}_{\mu}^{-1}\left[\frac{1}{\beta} T_{\mathbf{r}}^{\beta}\right]$,

where the function $T_{\mathbf{r}}^{\beta}$ can be related to the local propagator $C_{\mathbf{r}=\mathbf{r}^{\prime}}^{\beta}$ using the Bloch Equation (3):

$T_{\mathbf{r}}^{\beta}=-\left[\frac{\partial}{\partial \beta}+V(\mathbf{r})\right] C_{\mathbf{r}=\mathbf{r}^{\prime}}^{\beta}$

Thus, for a local potential $V(\mathbf{r})$ the kinetic energy density is given directly in terms of the local propagator. (In fact, Eq. (60) expresses the kinetic energy simply as the difference between the total and the potential energy.)

In order to calculate the kinetic energy part of (58), we do however not even need to know $\tau(\mathbf{r})$. In fact, it can be shown that for our approximations to the propagator and the densities derived from them, the virial theorem is fulfilled:

$\int \tau(\mathbf{r}) d^{3} r=\frac{1}{2} \int \mathbf{r} \cdot \boldsymbol{V} \boldsymbol{V}(\mathbf{r}) \rho(\mathbf{r}) d^{3} r$.

To prove this, we take the Laplace transform of (61):

$\int T_{\mathbf{r}}^{\beta} d^{3} r=\frac{1}{2} \int \mathbf{r} \cdot \boldsymbol{V} V(\mathbf{r}) C_{\mathbf{r}=\mathbf{r}^{\prime}}^{\beta} d^{3} r$.

For our propagators $C_{\mathbf{r}, \mathbf{r}}^{\beta^{(1)}}$ and $C_{\mathrm{r}, \mathrm{r}}^{\beta(2)}$ given above and the functions $T_{\mathbf{r}}^{\beta}$ derived from them by (60), it can be checked straightforwardly by partial integrations that (62) is fulfilled up to terms containing those higher order derivatives which are already neglected in $C_{\mathrm{r}, \mathrm{r}}^{\beta^{(1)}}$ and $C_{\mathbf{r}, \mathbf{r}}^{\beta^{(2)}}$. (For the "harmonized" approximation this is of course no surprise since it has the form of a harmonic oscillator propagator.)

With this, the total energy can be expressed directly in terms of the density $\rho(\mathbf{r})$ and the potential $V(\mathbf{r})$ :

$E=\int d^{3} r\left[V(\mathbf{r})+\frac{1}{2} \mathbf{r} \cdot \nabla V(\mathbf{r})\right] \rho(\mathbf{r})$.

We note in passing, that the same can also be done in the Thomas-Fermi approximation, since it is wellknown that the densities $\rho_{\mathrm{TF}}(\mathbf{r})$ and $\tau_{\mathrm{TF}}(\mathbf{r})$ also fulfill the virial theorem. The integral in (63) is in this case limited to the classically allowed region. 


\section{Connection with Other Approaches}

The connection of our results with the Wigner-Kirkwood expansion [1-4] is straightforward, keeping in mind that this expansion is equivalent to an expansion of $C_{\mathbf{r}, \mathbf{r}^{\prime}}^{\beta}$ around $\beta=0$. Thus, by a Taylor expansion of the propagator $C_{\mathbf{q}, \mathbf{s}}^{\beta^{(2)}}$ (54) in powers of $\beta$, keeping the classical propagator (9) as a factor in front, we obtain for $\mathbf{s}=0$ :

$$
\begin{aligned}
& C_{\mathbf{r}}^{\beta(\mathrm{WK})}=C_{\mathbf{r}}^{\beta(\mathrm{TF})}\left\{1-\frac{\mathbf{h}^{2}}{12 m} \Delta V(\mathbf{r}) \beta^{2}\right. \\
& \left.+\frac{\mathbf{h}^{2}}{24 m}[\boldsymbol{V} V(\mathbf{r})]^{2} \beta^{3}+\mathcal{O}\left(\mathbf{h}^{4}\right)+\cdots\right\} .
\end{aligned}
$$

This is just the Kirkwood-expansion including up to second derivatives of $V$. (Of course, the higher derivatives also come out with the correct coefficients, if they are included in $F$ and $G$ of (10) before the Taylor expansion.)

It is in this sense that our "harmonized" propagators (31), (54) represent a partial resummation of the Kirkwood-series (64) to all orders in $\mathbf{h}$, neglecting higher than second derivatives of $V$. The "linearized" solutions, Equations (25) and (46), result in the same way from a resummation of the first derivatives only. Mathematically, it is this resummation which makes the turning point problem disappear and creates densities with a well-behaved exponential tail, a fact which was recognized by Bhaduri [8].

An approach which is in some way related to what we call here the "linearized" approximation has been suggested by Balazs and Zipfel [18], although their derivation is quite different from ours. In fact, if we replace the derivative $V^{\prime}(q)$ in our expression (25) (for $s$ $=0$ ) by its value at the turning point, we obtain a form which is exactly equivalent to one of their approximations to the Wigner function $\left(f_{F}(E)\right.$ in $(10)$ of the first paper in [18]). For a strictly linear potential both approximations give thus the exact result. An extension of the approach of Balazs and Zipfel was recently used [19] and found to lead to well-behaved densities. The relation between this extension and our present work is, however, not obvious.

The approximation of Bhaduri [8] extends over the "linearized" form (46) by the inclusion of some selective higher order derivatives of the potential. This result can be obtained from our results by Taylor expanding both $\left|G^{(2)}\right|^{-1 / 2}$ using (29) and the exponent $F$ of (10) up to order $\beta^{3}$. This gives

$$
\begin{aligned}
& C_{\mathbf{q}, \mathbf{s}=0}^{\beta(\text { (h) }}=\left(\frac{m}{2 \pi \mathbf{h}^{2}}\right)^{3 / 2} \beta^{-3 / 2}\left[1-\frac{\mathbf{h}^{2}}{12 m} \beta^{2} \Delta V(\mathbf{q})\right] \\
& \exp \left\{-\beta V(\mathbf{q})+\frac{\mathbf{h}^{2}}{24 m} \beta^{3}\left[(V V(\mathbf{q}))^{2}-\frac{\mathbf{h}^{2}}{10 m} \Delta^{2} V(\mathbf{q})\right]\right\} .
\end{aligned}
$$

Contrary to our "harmonized" solution, the second order derivatives are here not consistently resummed. The form (65) was however shown in [8] to yield reasonable densities with the correct fall-off in the surface. It remains to be checked numerically in realistic potentials, how much is gained by the resummation of the second derivatives to all orders.

We have, however, reasons to believe that it is important to include some contributions from the local curvature of the potential. In fact, the results obtained with the Kirkwood-expansion in realistic nuclear potentials [7] have demonstrated that leaving out all second and higher derivatives may easily lead to errors of $\sim 50-100 \mathrm{MeV}$ in the average binding energies of heavy nuclei (see also in Section 4 below). Therefore, the second order derivatives of the potential may be quite important in determining the average nuclear surface properties.

In a recent paper [9], good results for the density in one dimension were obtained in developing the potential up to second order around the classical path. It turns out that our present approximation is contained in this theory; we therefore briefly want to show the connection between both approaches. The expression which was obtained in [9] is of the following form $(t=$ $-i \mathbf{h} \beta)$ :

$$
\begin{aligned}
& C_{x, x^{\prime}=q_{0}}^{t}=\left(\frac{m}{2 \pi \mathbf{h}^{2}}\right)^{1 / 2} \cdot \frac{e^{-\tilde{F}}}{\sqrt{\tilde{G}}}, \\
& \tilde{F}=-\frac{i}{\mathbf{h}}\left[W+p(x-q)+\frac{m}{2} \frac{\dot{G}}{\tilde{G}}(x-q)^{2}\right],
\end{aligned}
$$

where $q(t)$ is the classical path with initial conditions $q_{0}$ $=q(0) ; m \dot{q}(0)=p_{0}$. In these expressions $W$ is the classical action, $p=m \dot{q}$ is the momentum and $\tilde{G}$ obeys the following equation of motion

$m \frac{\partial^{2} \tilde{G}(t)}{\partial t^{2}}=-\left.\frac{\partial^{2} V}{\partial x^{2}}\right|_{x=q} \tilde{G}(t)$,

with the initial conditions $\tilde{G}(0)=0 ; \dot{\tilde{G}}(0)=i / \mathbf{h}$.

Equation (68) has exactly the same form as (28) above which determines our $G^{(2)}$. Indeed, if we keep only up to second derivatives of $V$, we can replace $V^{\prime \prime}(q)$ by $V^{\prime \prime}\left(q_{0}\right)$ in (68) and get back our expression $G^{(2)}(29)$. Developing in the same manner the classical path $q(t)$ and the action $W$ up to second order derivatives of $V$, we obtain from (67) the expression (30) for $F^{(2)}$. The same remains true also in three dimensions when one uses locally normal coordinates.

The full expression (66) gives for one dimensional potentials a better reproduction of the density fluctuations in the interior than our present approximation. This is, however, achieved at the expense of solving exactly the classical equation of motion, which 
for arbitrary potentials is not always easy, especially in three dimensions. Thus, our present approach can be taken as an approximation to the one of [9] which is easier to use and can be applied directly also in three-dimensional problems.

\section{Numerical Results}

In our numerical calculations of density distributions we chose to do the inverse Laplace transforms by a numerical integration using the formula [10]:

$\mathscr{L}_{\mu}^{-1}[f(\beta)]=\frac{1}{2 i \pi} \int_{c-i \infty}^{c+i \infty} e^{\mu \beta} f(\beta) d \beta$,

rather than programming the explicit formulae of the densities given above in the text. This has the advantage that one always must perform one summation only, also in the three-dimensional case, where the density e.g. in our "harmonized" approximation is given by a triple sum. Furthermore, the forms of our approximated propagators are much easier to compute than the Airy or Parabolic Cylinder functions with their complicated arguments.

Care has to be taken to get the optimal convergence of the integral (69) with respect to the choice of the constant $c$ which is an arbitrary real positive number, and the limits of the interval in $\operatorname{Im} \beta$. (If the argument $f(\beta)$ in $(69)$ has infinitely many poles in the right half of the complex $\beta$-plane, as is the case for our result (31) if $V^{\prime \prime}(q)<0$, then $(69)$ is actually the inverse of a two-sided Laplace-transform; $c$ must then lie within the first strip with $\operatorname{Re} \beta>0$ within which $f(\beta)$ is analytic.)

In all our results given below, the integration in (69) was done along a straight line parallel to the imaginary $\beta$ axis with $c=0.1 \mathrm{MeV}^{-1}$. We chose a numerical accuracy of the densities at each point of $10^{-4}$. With that, an interval $0 \leqq \operatorname{Im} \beta \leqq 15 \mathrm{MeV}^{-1}$ was sufficient (using the symmetry of the integrand); the mesh size $\Delta \beta_{i}$ was kept variable according to the local behaviour of the integrand: for small values of the variable $\mathbf{q}$, a typical mesh size was $A \beta_{i}=(3$ to 6$) \cdot 10^{-3} \mathrm{MeV}^{-1}$, a larger mesh was sufficient for higher values of q. Higher accuracies than $10^{-4}$ could easily have been obtained (also with smaller values of $c$ ) at the cost of more computer time.

As we mentioned in Section 2.1, we applied a Strutinsky-smoothing to our "harmonized" propagator $C_{\mathbf{q}, \mathbf{s}}^{\beta(2)}$ (31), in order to avoid discontinuities in the interior of the nucleus $\left(V^{\prime \prime}(q)>0\right)$. In energy space, the Strutinskysmoothing is usually done by introducing average occupation numbers $\tilde{n}_{i}[16,20]$ (which would replace the step functions in (34)). In $\beta$-space, this is equivalent to multiplying the propagator by a factor [4]

$\Gamma(\gamma, \beta)=e^{\frac{1}{2} \gamma^{2} \beta^{2}}\left\{1-\frac{1}{4} \gamma^{2} \beta^{2}+\frac{1}{32} \gamma^{4} \beta^{4}-\cdots\right\}$.

(The terms in brackets correspond to the curvaturecorrections $[4,20]$.).

In usual Strutinsky averaging $[16,20]$ with harmonic oscillator-like potentials, the smoothing width $\gamma$ must be chosen to be $\gamma \gtrsim \mathbf{h} \omega$ to get unique results. This has also been checked for the density distributions of the harmonic oscillator potential $[16,4]$. The appropriate choice of $\gamma$ in our calculations is thus a value proportional to the local frequency $d(32)$ :

$\gamma=a_{\gamma} d=a_{\gamma} \sqrt{\frac{\mathbf{h}^{2}}{m}\left|V^{\prime \prime}(q)\right|}$

with

$a_{\gamma} \approx 1-2$.

In our calculations in one dimension we found that a variation of the proportionality constant $a_{y}$ in the range (72) left the resulting densities unchanged within much less than $1 \%$ at all points (where $V^{\prime \prime}(q)>0$ ).

In three-dimensional potentials, one has to distinguish between the different local normal frequencies $\omega_{i}(53)$. In the spherically-symmetric case, for which we present a preliminary result below, the expression (54) of the propagator $C_{\mathbf{q}, \mathbf{s}}^{\beta(2)}$ reduces for $s=0$ to

$C_{r, r}^{\beta(2)}=\left(\frac{m}{2 \pi \mathbf{h}^{2}}\right)^{3 / 2}\left[G_{r}^{(2)}\right]^{-1 / 2} e^{-F_{r}^{(2)}}$,

where $F_{r}^{(2)}$ has the same form as (30) for $s=0$, but taking the derivatives $V^{\prime}(r)$ and $V^{\prime \prime}(r)$ with respect to the radial variable, and $G_{r}^{(2)}$ is given by

$G_{r}^{(2)}=\frac{1}{\mathbf{h}^{3} \omega_{r} \omega_{\perp}^{2}} \operatorname{Sinh}\left(\beta \mathbf{h} \omega_{r}\right)\left[\operatorname{Sinh}\left(\beta \mathbf{h} \omega_{\perp}\right)\right]^{2}$

with the two frequencies

$\omega_{r}=\sqrt{\frac{1}{m} V^{\prime \prime}(r)}, \quad \omega_{\perp}=\sqrt{\frac{1}{m r} V^{\prime}(r)}$.

A sufficient smoothing is in this case obtained taking

$\gamma=a_{\gamma} \max \left(\mathbf{h} \omega_{r}, \mathbf{h} \omega_{\perp}\right)$.

This Strutinsky-smoothing has not only the effect of consistently averaging the density, but also the convenient advantage that it speeds up the numerical integration. In fact, the exponential in (70) acts as a Gaussian along the path of integration and thus damps the integrand away from the real axis. As a result, the range of integration could be cut down by a factor of 
$\sim 3$ to 15 , depending on the position $\mathbf{r}$, still obtaining the same numerical accuracy.

Since this is a desirable effect and since it has been shown [16] that a Strutinsky-averaging of exact densities does not change them at all in the tail region, we also applied the smoothing factor (70) in the outside region where $V^{\prime \prime}(r)<0$, using (71) in the one-dimensional and $\gamma=a_{\gamma} \cdot \mathbf{h} \omega_{\perp}$ in the three-dimensional case. We verified that here, too, the results of the exact numerical integration were not changed if a factor $a_{\gamma} \approx 0.5-1.5$ was used. We emphasize that this smoothing in the tail region is a pure matter of convenience. It does not affect the results within the accuracy of the numerical integration, but reduces the computation time by more than a factor of 10 .

As an example of a finite-depth potential in one dimension we chose the so-called "Eckardt-Potential"

$V(x)=\frac{V_{0}}{[\operatorname{Cosh}(x / a)]^{2}}$,

whose eigenfunctions and eigenvalues are known analytically (see, e.g. Ref. [11]). For convenience we used dimensionless variables, choosing $V_{0}=-50$ and $a$ $=1, \mathbf{h}^{2} / m=1$.

In Figure 1 we present the density distributions for three different numbers of particles. (With our choice of constants, a maximum of $n=10$ particles can be accommodated in the well (77).) The solid lines are the exact densities. The dashed lines are the results for our Strutinsky-smoothed harmonic approximation $\rho^{(2)}(x)$, and the dashed-dotted lines represent the ThomasFermi results $\rho^{(\mathrm{TF})}(x)$. We see that the approximation $\rho^{(2)}(x)$ leads to a well-defined surface and a reasonable fall-off in the tail region. No irregularity is left at the turning point where $\rho^{(\mathrm{TF})}(x)$ goes to zero with an infinite slope. In the interior, we obtain some quantum oscillations. They have approximately the same amplitude as the ones in the exact densities, but they are different in wavelength and phase.

In Figure 2 we present for the same cases the "linearized" approximation $\rho^{(1)}(x)$ (dashed lines) alongside the exact densities (solid lines). In the surface and tail regions, the results for $\rho^{(1)}(x)$ are very similar to those of $\rho^{(2)}(x)$ in Figure 1. In the interior, the exactly inverted densities $\rho^{(1)}(x)$ which are given by (41) and (43) (with $s=0$ ), have oscillations that get more and more rapid, but with a decreasing amplitude, as one approaches the centre $x=0$. This is shown in the figure by the dotted parts of the curves. This feature is easily understood from the nature of the Airy functions in (41) and (43) and the fact, that the scaling function $\sigma(x)(42)$ diverges as $x$ (and with it $V^{\prime}(x)$ ) goes to zero. Thus, the asymptotic oscillations of the Airy function $\mathbf{A i}(z)$ (for $z \rightarrow-\infty)$ are all concentrated at $x=0$. Since these

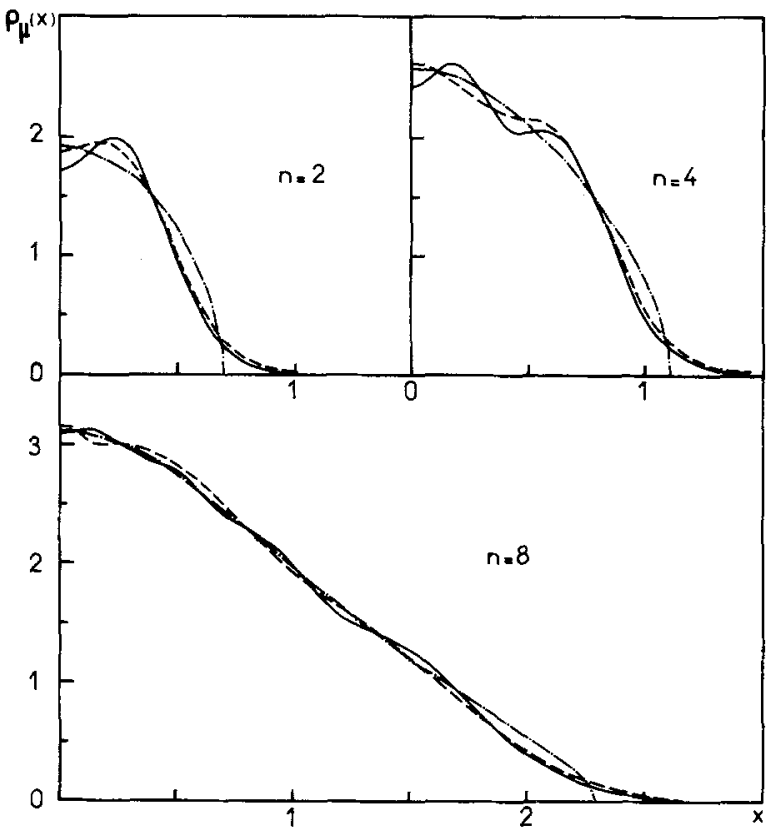

Fig. 1. Density distributions for 2,4 and 8 particles in the onedimensional Eckardt potential (77). The solid lines are the exact densities $p(x)$, the dashed lines are the Strutinsky-smoothed "harmonized" densities $\rho^{(2)}(x)$. Dash-dotted lines are the Thomas-Fermi results

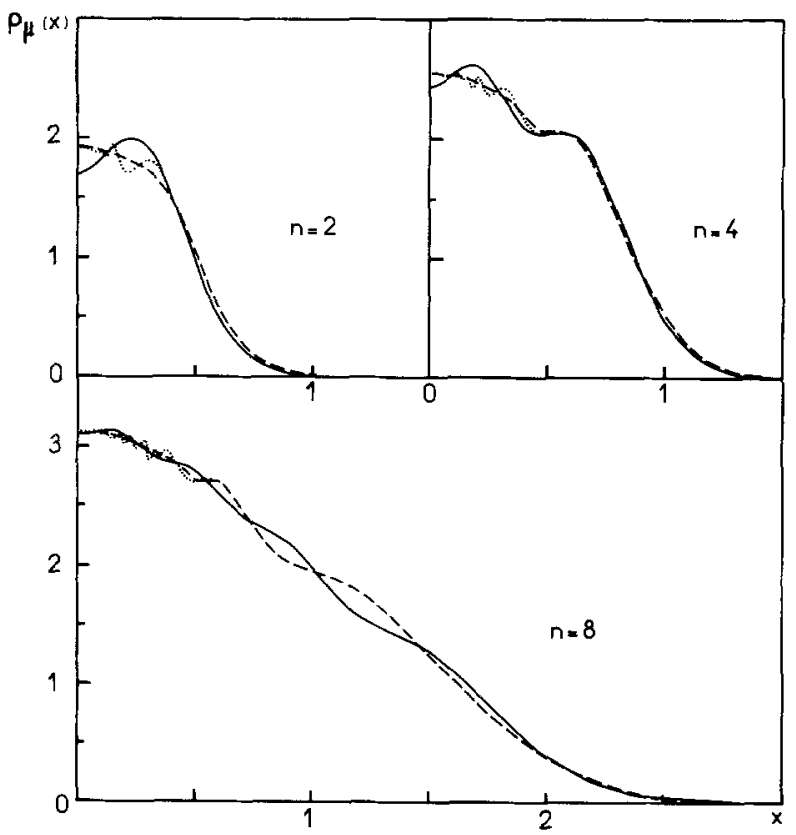

Fig. 2. As in Figure 1; the dashed lines are here Strutinsky-smoothed "linearized" densities $\rho^{(1)}(x)$. Near the origin, the dotted parts of the curves correspond to the unsmoothed results $\rho^{(1)}(x)$ which otherwise are identical with the smoothed ones. (TF densities not shown here)

oscillations are completely spurious, we have averaged them out, again using a Strutinsky-smoothing factor with the definitions (70)-(72). The result is shown by the dashed parts of $\rho^{(1)}(x)$, which become smooth and approach the TF-densities (see Fig. 1) close to $x=0$. 


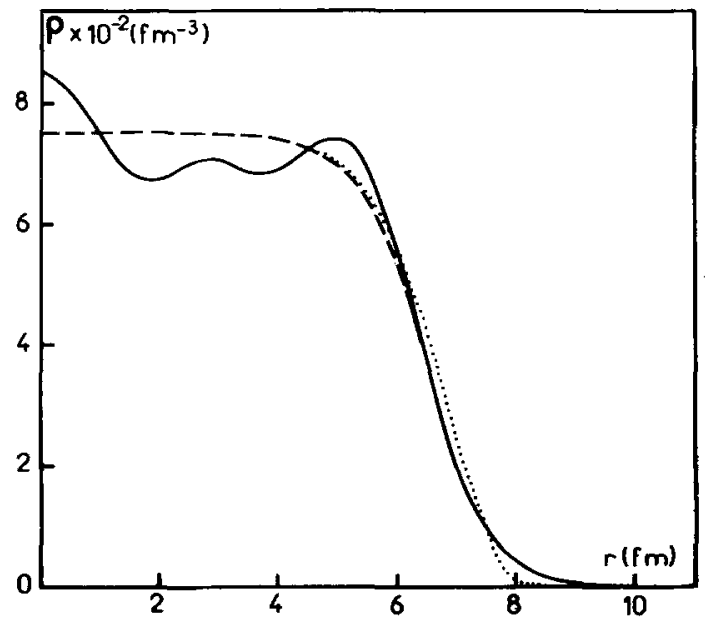

Figure 3. Density distributions for $A=184$ particles in a spherical Woods-Saxon potential, Equation (78). The solid line is the exact density; the dotted line is the result $\rho^{(1)}(r)$ (not smoothed) which for $r \lesssim 4.5 \mathrm{fm}$ coincides with the approximation $\rho^{(2)}(r)$. The latter (dashed line) agrees with the exact density for $r \gtrsim 6.5 \mathrm{fm}$. The Thomas-Fermi density (not explicity shown) coincides with the "linearized" one $\rho^{(1)}(r)$ up to $\sim 8 \mathrm{fm}$; at the turning point $r_{\lambda} \simeq 8.2 \mathrm{fm}$ it goes to zero with a zero slope

In both approximations we get thus reasonably wellbehaved densities. The quantum oscillations do not correctly reproduce the exact ones and should therefore not be given much physical significance. The surfaces of the densities $\rho^{(1)}$ and $\rho^{(2)}$ are, however, rather close to the exact ones, and have a reasonable exponential tail. The fact that apparently not much is changed in going from the linearized to the harmonized approximation, is particular to the one-dimensional case.

In Figure 3 we show the results for a three-dimensional spherical Woods-Saxon-potential:

$V(r)=\frac{V_{0}}{1+\exp \left(\frac{r-R_{0}}{a}\right)}$,

using the constants $V_{0}=-44 \mathrm{MeV}, a=0.67 \mathrm{fm}, R_{0}$ $=1.27 \mathrm{fmA}^{1 / 3}$ and $\mathbf{h}^{2} / m=41.49 \mathrm{MeVfm}^{2}$. The densities shown correspond to $A=2 N=184$ particles which in the exact case is a closed-shell configuration. The exact density is again shown by the solid line (it was obtained from solving exactly the Schrödinger equation with the program of [16] and summing the wavefunctions). The linearized density $\rho^{(1)}(r)$ is shown by dots and the Strutinsky-smoothed harmonized density $\rho^{(2)}(r)$ by the dashed line. In the interior $(r \lesssim 4 f m)$ both approximations go over into the Thomas-Fermi density. No oscillations can be seen in $\rho^{(1)}(r)$ near $r=0$, as it was the case in one dimension without Strutinsky-smoothing. The Thomas-Fermi density (not shown in Fig. 3) follows almost indistinguishably the density $\rho^{(1)}(r)$ over the entire region, except very close to the turning point $r \simeq 8.2 \mathrm{fm}$, where it goes to zero with a zero slope.
The surprising result is, however, that the harmonized density $\rho^{(2)}(r)$ approaches the exact one already at $r \simeq 6.5 \mathrm{fm}$ and cannot be distinguished from it within the accuracy of the drawing for the whole outside region. We see thus, that in this three-dimensional case, an important improvement is reached in the harmonic approximation as compared to the linear one, which here is practically identical to the Thomas-Fermi case except for a tiny exponential tail outside $r \approx 8.2 \mathrm{fm}$.

We have thus obtained an approximation $\rho^{(2)}(r)$ which in the far inside of the nucleus goes over to the ThomasFermi density and in the outer surface coincides with the exact density, leading to a well-defined and realistic surface region. This density and the corresponding kinetic energy density $\tau^{(2)}(r)$ may thus be used directly in a variational approach in order to calculate nuclear binding energies with Skyrme-type forces.

As a first step in this direction which allows to check the kind of energies that are obtained with our approximation, we calculated the energy $E(63)$, which for the exact density equals the sum of occupied levels, for the above-mentioned one body potentials.

In the one-dimensional Eckardt-potential, Equation (77), the resulting energies are not very instructive. Here even the Thomas-Fermi approximation leads to very good results, the relative difference between the corresponding energies $E^{\mathrm{TF}}$ and the exact ones, $E^{\mathrm{ex}}=\sum_{i=1}^{N} \varepsilon_{i}$, being only $\sim 0.2 \%$ for all numbers of particles $(1 \leqq N \leqq 10)$. In going to the harmonized approximation $\rho^{(2)}(x)$, this difference is further reduced, but not much can be learned from this result. This is not so surprising, since on one hand, there is not much shell structure in the energy levels of a one-dimensional potential and thus their sum can always well be approximated by a smooth functional, and on the other hand the semiclassical corrections are very small in this case.

For a realistic three-dimensional potential, the calculation of the total energy is more interesting. As preliminary results for a spherical case, we show in Table 1 the energies obtained in the different approximations for the Woods-Saxon potential (78) with the same parameters as above. The first three columns

Table 1. Total energies (in MeV) for 96 and 184 particles in a spherical Woods-Saxon potential (see Eq. (78)). $E^{\mathrm{TF}}, E^{(1)}$ and $E^{(2)}$ are obtained through (63) using the Thomas-Fermi, the "linearized" and the "harmonized" approximations to the density, respectively. $\tilde{E}$ is the Strutinsky-averaged result $[16,20]$ and $E^{\text {exact }}$ the exat sum of occupied levels. The accuracy is $\sim 1 \mathrm{MeV}$ in $E^{(2)}$ and $\tilde{E}$ (see text) and better than $0.1 \mathrm{MeV}$ in the other results

\begin{tabular}{rlllllr}
\hline$A=2 N$ & $E^{\mathrm{TF}}$ & $E^{(1)}$ & $E^{(2)}$ & $\tilde{E}$ & \multicolumn{1}{l}{$E^{\text {exacl }}$} & $\delta E$ \\
\hline 96 & -1872.7 & -1873.2 & -1824 & -1811 & -1786.6 & 24.4 \\
184 & -3785.8 & -3786.7 & -3724 & -3689 & -3681.8 & 7.2 \\
\hline
\end{tabular}


show the energies (63) obtained with the ThomasFermi, the "linearized" and the "harmonized" approximations to the density, respectively. We see that almost nothing is changed if one goes from the TFresult $E^{(\mathrm{TF})}$ to the linearized approximation $E^{(1)}$. This could be expected from our resulting densities $\rho^{(1)}(r)$ (see Fig. 3) which were almost identical to the $T F$ ones. However, in going to the "harmonized" approximation, i.e. by summing up all contributions from the second derivatives of the potential, the total energy increases by $\sim 49 \mathrm{MeV}$ for $A=96$ and by $\sim 63 \mathrm{MeV}$ for $A=184$ particles. Thus, appreciable contributions to the energies are gained in this approximation. As we mentioned above, we applied a Strutinsky-smoothing to the density $\rho^{(2)}(r)$. In varying the averaging width $\gamma$ and the order of the curvature-corrections (i.e. the number of terms in brackets in (70)), we found that the typical "plateaux" were present in the functions $E^{(2)}(\gamma)$ for values of $a_{y}$ in (71) of $\sim 1.0-1.6$, as they are present in the usual Strutinsky-averaged energies for a WoodsSaxon potential [20]. The energies $E^{(2)}$ are thus welldefined within less than $\sim 1 \mathrm{MeV}$.

In Table 1 we show also the energies $\tilde{E}$ obtained with the usual Strutinsky method, i.e. by averaging the exact spectrum of the potential. (Here, too, the plateau-values are converged within less than $\sim 1 \mathrm{MeV}$.) For completeness, the exact sums of the occupied levels, $E^{\text {exact }}$, and the shell-corrections, defined by $\delta E=E^{\text {exact }}-\tilde{E}$, are given in the last two columns.

The quantities $E^{\text {exact }}$ and $\delta E$ are just given here for illustration; the relevant energies to which our semiclassical results have to be compared, are the quantities $\tilde{E}$. We know from earlier work [7] that the Strutinskyaveraged energies $\tilde{E}$ agree within $\sim 1-2 \mathrm{MeV}$ with the energies obtained in the ETF-model by summing the Wigner-Kirkwood series up to order $\mathbf{h}^{4}$. Our results $E^{(2)}$ lie below the values of $\tilde{E}$ by an amount of $13 \mathrm{MeV}$ for $A=96$ and $35 \mathrm{MeV}$ for $A=184$. Since all the essential contributions to the ETF-energies should be contained in our approximation (the terms proportional to $\mathbf{h}^{4}$, which contain higher than second derivatives of $V(r)$, contribute less than $\sim 2 \mathrm{MeV}[4,7]$ ), these differences seem to indicate that some cancellations between higher powers of first and second derivatives on one hand and the higher than second derivatives on the other are taking place in the Kirkwood expansion, thus leading to a better convergence of the latter, compared to our "harmonized" approximation. However, since the results presented here are preliminary ones, we cannot draw definite conclusions on the difference between $E^{(2)}$ and $\tilde{E}$ yet. For that, more systematical studies have to be done, which we will present in the near future.

Another point which we are investigating is the question, whether the Strutinsky-smoothing applied to $\rho^{(2)}(r)$ has not spoiled too much information. Clearly, we have obliterated all the quantum fluctuations in the interior of the nucleus. In principle the unsmoothed densities $\rho^{(2)}(r)$, although having discontinuities in the interior, can be integrated to obtain the total energy; for that, a very fine mesh of integration in the $r$ direction is necessary, thus requiring more computer time. (For the present calculations, we used a mesh with $\Delta r_{i}=0.05 \mathrm{fm}$, leading to a total computing time of $\sim 30$ seconds on a CDC -6600 for one density distribution and the corresponding energy $E^{(2)}$.) We are also studying another method of smoothing, which may preserve some of the density fluctuations and still leads to continuous densities. Finally, the inclusion of terms containing higher than second derivatives of $V(\mathbf{r})$ is investigated, too.

Our main conclusions at present are: 1 . We have an approximation which yields densities that are welldefined everywhere in space and have the right exponential fall-off, starting already at the half-density radius. 2 . In three-dimensional cases the contributions of the second derivatives of the potential are very important both for obtaining the correct surface behaviour of the density and for obtaining the main part of the semiclassical corrections to the total energy. The "linearized" approximation, containing only first derivatives of $V$, leads to densities which do have an exponential tail but otherwise are practically identical to the $T F$ densities and lead also to the uncorrected $T F$ energies.

The applications to deformed potentials are straightforward as soon as the eigenvalue problem (49) is numerically solved, and will be presented in a forthcoming publication.

\section{Summary and Discussion}

We have developed an approximation scheme for the density matrix of independent Fermions in a local potential $V$. Generalizing the Thomas-Fermi method in which the potential is held locally constant, we proceed by successively including first and second derivatives of $V$ in locally linearizing and harmonizing the potential, respectively. It turns out that at both steps one can sum all the contributions and thus obtain closed expressions for the density matrix. The inclusion of contributions from higher than second derivatives of $V$ is straightforward using the series expansions of $G$ and $F$ in the propagator $(10)$; we have however not succeeded to sum all contributions of the third derivatives. This is not surprising, since a closed expression up to third order would mean an exact solution for the cubic potential $V(r)=a r^{3}$, which does not exist. We have shown that our results correspond to partial resummations of the Wigner-Kirkwood series, neglecting the higher derivatives of $V$. 
Our resulting density $\rho^{(2)}$ is a function of the potential and its first and second derivatives; it is exact for linear and quadratic potentials. Its main feature is that it is well-defined everywhere in space, including the classical turning point, and has the correct exponential fall-off. In fact, for a spherical three-dimensional Woods-Saxon potential, the density $\rho^{(2)}(r)$ is in excellent numerical agreement with the exact density in the whole surface region outside the half-density radius. In the linearized approximation, where only the first derivatives of the potential are retained, the density $\rho^{(1)}(r)$ is for the same potential almost identical with the Thomas-Fermi density, except for a tiny exponential tail outside the turning point.

In the interior region, the densities $\rho^{(2)}(r)$ have discontinuities which come from the locally harmonic approximation (with a positive curvature $V^{\prime \prime}(r)$ ) leading to a position-dependent eigenvalue spectrum. In order to obliterate these spurious fluctuations, we applied a local Strutinsky-smoothing to $\rho^{(2)}(r)$, leading to perfectly smooth functions which approach the ThomasFermi results in the interior of the nucleus. It will be investigated whether another way of smoothing allows to retain some of the quantum fluctuations in the density.

Swiatecki [21] investigated what we call here the linearized case; a related approach which includes first derivatives of $V$ is that of Balazs et al. $[18,19]$. Otherwise we are not aware of any continuation of these approaches except the ansatz of Bhaduri [8] who summed up all first derivatives and included some contributions from higher derivatives of $V$.

We have demonstrated that the inclusion of the second derivatives is important for the total energy of threedimensional systems. The linearized approximation gives practically the same energy as the Thomas-Fermi model, thus missing important contributions to the surface energy. In this sense we believe to have achieved the first - at least partially - successful attempt to both remove the turning point problem of the ETF model and include the main semiclassical corrections to the energy. We should stress that we have obtained closed expressions not only for densities, but for the whole (nonlocal) density matrix. We shall in the near future investigate numerically the off-diagonal behaviour of our results; since the latter is already quite good in the Thomas-Fermi case, we can hope that it will be very well represented by our expressions, where the dependence on the relative coordinate $\mathbf{s}$ is included up to second order. It should also be straightforward to extend our approximation to nonlocal potentials, tak-

Note Added in Proof: The differences between $E^{(2)}$ and $\tilde{E}$ in Table 1 are appreciably reduced if one does not use the virial theorem, but Equation (57) or (59) to calculate $\tau(\mathbf{r})$.

After this paper had been finished, our attention was drawn to the work of R. Baltin [Z. Naturforschung 27a (1972) 1176], who has ing into account e.g. an effective mass or a spin-orbit contribution.

An interesting field of applications would certainly be the study of tunnelling and scattering processes (e.g. also heavy ion reactions). In this respect it is interesting to note that our resummation procedure using the propagator gives besides the right initial conditions in time also the apparently correct boundary conditions in space. As can be seen from our results in the outside region of a three-dimensional potential, the application of our procedure to tunnelling processes is expected to be especially well suited.

The authors are indebted to B.K. Jennings for very stimulating discussions.

\section{References}

1. Wigner, E.: Phys. Rev. 40, 749 (1932)

2. Kirkwood, J.G.: Phys. Rev. 44, 31 (1933)

3. Bhaduri, R.K., Ross, C.K.: Phys. Rev. Lett. 27, 606 (1971)

4. Jennings, B.K.: Ph. D. Thesis, McMaster University, 1976

5. Bohigas, O., Campi, X., Krivine, H., Treiner, J. : Phys. Lett. 64 B, 381 (1976)

6. Chu, Y.H., Jennings, B.K., Brack, M.: Phys. Lett. 68 B, 407 (1977)

7. Jennings, B.K., Bhaduri, R.K., Brack, M.: Nucl. Phys. A 253, 29 (1975) and Phys. Rev. Lett. 34, 228 (1975)

8. Bhaduri, R.K.: Phys. Rev. Lett. 39, 329 (1977)

9. Gumpertsberger, G., Schuck, P.: Phys. Lett. 66 B, 219 (1977)

10. Abramowitz, M., Stegun, I.A.: Handbook of Mathematical Functions, fifth printing, New York: Dover 1968

11. Krivchenkov: Collected Problems in quantum mechanics, Ed. Ter Haar, D., London: Infosearch 1964

12. Voros, A.: Thèse d'Etat, Saclay 1977

13. Feynman, R.P., Hibbs, A.R.: Quantum Mechanics and Path Integral, New York: McGraw Hill 1965

14. Erdelyi, A. (Editor): Higher Transcendental Functions, Vol. II, New York: McGraw Hill, 1963; eqs. 8.5 (11) and 10.13 (22)

15. Strutinsky, V.M.: Nucl. Phys. A 95, 420 (1967); A 122, 1 (1968)

16. Brack., M., Damgaard, J., Jensen, A.S., Pauli, H.C., Strutinsky, V.M., Wong, C.Y.: Rev. Mod. Phys. 44, 320 (1972)

17. Vautherin, D., Brink, D.M.: Phys. Rev. C 5, 626 (1972); see also Negele, J.W., Vautherin, D.: Phys. Rev. C 5, 1472 (1972), and ibid. C 11, 1031 (1975)

18. Balazs, N., Zipfel, G.: Ann. Phys. (New York) 77, 139 (1973); J. Math. Phys. 15, 2086 (1974)

19. Balazs, N., Pauli, H.C.: Preprint Heidelberg, MPIH-1977-V9

20. Brack, M., Pauli, H.C.: Nucl. Phys. A 207, 401 (1973)

21. Swiatecki, W.J.: Proc. Phys. Soc. (London) A 65, 285 (1955)

M. Durand

Institut des Sciences Nucléaires (IN2 P3-U.S.M.G.)

BP 157 Centre de Tri

F-38042 Grenoble Cedex

France

M. Brack

P. Schuck

Institut Max von Laue-

Paul Langevin

BP 156 Centre de $T_{r i}$

F-38042 Grenoble Cedex

France

treated the linearized approximation, and that of D.H.E. Gross [Phys. Lett. 42B (1972) 41], who derived the harmonized form (48, 31 ) of the propagator in the local case $(s=0)$, but did not calculate the corresponding density distributions. 